\title{
Reproducibility of vaginal immobilization balloons in situ overnight for cervical cancer brachytherapy
}

\author{
Uma D. Goyal, MD**, Paras P. Mehta, BA*, Susan Samreth, MD², John Gloss, MS³, Haiyan Cui, PhD4 , Denise Roe, PhD4, \\ Shona Dougherty, MB, ChB, PhD' \\ *Uma D. Goyal and Paras P. Mehta contributed equally to this work. \\ 'Department of Radiation Oncology, University of Arizona College of Medicine, Tucson, USA, ${ }^{2}$ Department of Pediatrics, Eastern Virginia \\ Medical School, Norfolk, USA, ${ }^{3}$ West County Radiological Group, St. Lovis, USA, ${ }^{4}$ University of Arizona Mel and Enid Zuckerman College \\ of Public Health, Tucson, USA
}

\begin{abstract}
Purpose: The use of vaginal immobilization balloons placed into the vagina for immobilization of tandem and ovoid $(\mathrm{T}+\mathrm{O})$ applicator during high-dose-rate (HDR) brachytherapy delivery has been used at our institution, and seems to have improved our patient comfort, decreased procedure time, and minimized applicator misplacement. We aimed to show that these balloons, while originally marketed for single-day use, are safe and maintain applicator positioning/dosimetry when left in situ overnight for treatment delivery on sequential days.

Material and methods: Forty-two paired computed tomography (CT) scans from thirteen patients who underwent $\mathrm{T}+\mathrm{O}$ HDR treatments on sequential days with vaginal immobilization balloons in situ overnight were retrospectively compared to calculate mean change of balloon volumes and balloon/ $\mathrm{T}+\mathrm{O}$ distance to bony landmarks. Dosimetric planning was retroactively performed on day 2 using CT scan of each pair, and the change in estimated radiation delivery to the bladder and rectum was compared.

Results: No statistically significant overnight changes were found in balloon volumes or anterior balloon positioning. The posterior balloon shifted $-0.29 \pm 0.46 \mathrm{~cm}(p=0.03)$ to the anterior public symphysis and $0.32 \pm 0.50 \mathrm{~cm}(p=0.01)$ to the right femoral head. The tandem shifted $0.37 \pm 0.39 \mathrm{~cm}(p=0.002)$ to the pubic symphysis. There was no significant difference found in radiation delivered to the bladder or rectum between the paired scans.

Conclusions: This study showed minimal change in balloon volumes, balloons/ $\mathrm{T}+\mathrm{O}$ positioning, or in radiation dose to bladder and rectum when the applicator remained overnight. These findings support that inflatable vaginal immobilization balloons remaining in situ overnight for additional HDR T+O treatments on sequential days, is safe and provides stable dosimetry.

J Contemp Brachytherapy 2021; 13, 3: 280-285 DOI: https://doi.org/10.5114/jcb.2021.106117
\end{abstract}

Key words: tandem, ovoid, brachytherapy, balloons, overnight.

\section{Purpose}

Cervical cancer is the fourth most common cancer in women worldwide and is the leading cause of cancerrelated death for women in Eastern, Western, Middle, and Southern Africa [1]. In the U.S., the cases of cervical cancer in young females have decreased after the use of human papilloma virus vaccine [2]. The estimated number of cervical cancer patients in 2015 was approximately 12,900 cases [3].

In locally advanced cases of cervical cancer, chemotherapy combined with radiation therapy (RT) remains the current standard of care [4]. External RT and intracavitary brachytherapy delivered via tandem and ovoids $(\mathrm{T}+\mathrm{O})$ or tandem and ring, have long been the mainstay of the treatment regimen [5]. Both the above-mentioned RT modalities are generally recommended to maximize local and regional tumor control, and studies have shown decreased survival rates in patients where the brachytherapy is omitted [5-8].

Historically, based on the Manchester system, the location at which the prescription dose of brachytherapy radiation was defined, is known as point " $\mathrm{A}$ " located $2 \mathrm{~cm}$ superior to the vaginal fornices along the tandem and $2 \mathrm{~cm}$ perpendicularly lateral to the tandem [9]. This point also was commonly thought to describe where the ureters cross the uterine artery, which was found to be a point that limited toxicities due to findings of necrosis at the paracervical vessels, making it vulnerable to potential changes in dosimetry [10]. Studies have shown that there
Address for correspondence: Uma D. Goyal, Department of Radiation Oncology, University of Arizona, 3838 N Campbell Ave, Bldg. 2 Tucson, AZ 85719, USA, phone: +1 520-694-7236, e-mail: ugoyal@email.arizona.edu
Received: 01.12 .2020

Accepted: 23.02 .2021

Published: 13.05 .2021 
is a positive correlation between bladder dose, rectal dose, urinary bladder, and rectal complications, and severity of complications [11]. Therefore, it is vital to weigh the cost-benefit ratio of achieving a high-rate of cure and RT complications, which may at times significantly affect quality of life.

To limit the RT exposure to organs at risk (OARs), the current practice is to insert lubricated strips of gauze or other packing material into the space between the applicator and the anterior and posterior vaginal walls. This fixes the applicator and displaces the bladder and rectum anteriorly and posteriorly, respectively, thus reducing the overall dose delivered to these organs [12].

Malaker et al. [13] described a technique to reduce urinary bladder dose without compromising dose to the cervix by placing an inflated Foley catheter balloon between tandem source and base of the bladder. Eng et al. [14] developed another technique, in which an uninflated Foley balloon was inserted transvaginally above and below the tandem flange and then secured in place with vaginal packing. In this study, there was an average dose reduction of $7.2 \%$ and $9.3 \%$ to $D_{0.1 c c}$ of the bladder and rectum, respectively [14]. Alternatively, for patients with smaller volume vaginal cavities, Rai et al. [15] described a method, in which a bladder-rectum spacer balloon, a disposable latex balloon abutting the bladder and rectum and adjoined at the middle, demonstrated statistically significant dose reductions to the rectum compared to gauze packing technique.

At our center, the $\mathrm{T}+\mathrm{O}$ applicator used to deliver HDR brachytherapy is usually positioned with vaginal immobilization balloons anteriorly and posteriorly, to displace the bladder and rectum away from the radiation source. The $\mathrm{T}+\mathrm{O}$ applicator and balloons were left in situ overnight with the patient on strict bed rest, to deliver 3 fractions of brachytherapy. Preliminary data have shown minimal change in immobilization balloon volume while in situ overnight [16]. This is the first study to our knowledge looking at the overnight change of these balloons. In this study, we aimed to show that cervical balloon use for patients being treated with HDR $\mathrm{T}+\mathrm{O}$ brachytherapy is safe and reproducible for planning and treatment delivery, when left in situ overnight for treatments given on sequential days.

\section{Material and methods}

\section{Patients}

This retrospective study was approved by our institutional review board (IRB). Eligible patients included females over 18 years of age with locally advanced cervical cancer, undergoing $\mathrm{T}+\mathrm{O}$ brachytherapy delivery on sequential days. This technique was adopted as our program changed from low-dose-rate cesium-137 to HDR iridium-192, and continued to serve patients who traveled from long distances or rural regions for their brachytherapy in an academic setting.

We reviewed $44 \mathrm{CT}$ simulations of 22 paired treatments in 13 patients. The anterior balloon deflated in one of these paired treatments and was omitted from data analysis, leaving 42 CT simulations of 21 paired treatments in 13 patients.

\section{Procedure}

The $\mathrm{T}+\mathrm{O}$ Fletcher suit applicator (Varian Medical Systems, Palo Alto, CA, USA) was placed in the operating room under general anesthesia. Two immobilization balloons (Radiadyne Alatus, Latham, NY, USA) were then placed, with one arranged anteriorly between the ovoids and bladder, and one placed posteriorly between the ovoids and rectum. The balloons were then inflated in $10 \mathrm{cc}$ of air increments up to $40 \mathrm{cc}$ as patient anatomy allowed with continual checking of positioning of the balloons during inflation. A CT simulation $(\operatorname{sim} 1)$ was then performed in the immediate post-op period to determine the positioning of $\mathrm{T}+\mathrm{O}$ and balloons, allowing for dosimetric planning of the HDR treatments. Prior to sim 1, the Foley was clamped and the bladder was instilled with 30 cc of normal saline and contrast. After contouring and treatment planning (Eclipse BrachyVision version 11.0.47, Varian Medical Systems, Palo Alto, CA, USA), the plan was evaluated and approved by the treating physician and medical physicist. The patients then received their first of 3 brachytherapy treatments over 2 sequential days.

After delivery of the first fraction, the patient stayed in the hospital overnight with the $\mathrm{T}+\mathrm{O}$ applicator and balloons in situ. A second CT simulation ( $\operatorname{sim} 2)$ was performed the next morning prior to the second fraction, to ensure that no major movement of the applicator or balloons had occurred. Prior to sim 2, $30 \mathrm{cc}$ of normal saline was instilled into the bladder. The patient received the second fraction, and after the third fraction (all fractions were minimum 6 hours apart), the $\mathrm{T}+\mathrm{O}$ applicator and balloons were removed and the patient discharged. The patient returned a week later to receive a second $\mathrm{T}+\mathrm{O}$ implant, with again 3 fractions of HDR separated by a minimum of 6 hours, with fractions 2 and 3 being delivered the following day of implant. Each patient, therefore, had two T+O implants, with a total of 4 CT sim scans $(2 \times \operatorname{sim} 1$ and $2 \times \operatorname{sim} 2)$ and 6 HDR fractions, which were available for analysis.

\section{Dosimetry}

The paired simulations ( $\operatorname{sim} 1$ and sim 2) were accessed retrospectively, and primary outcomes including measurements of applicator positioning and balloon volumes were obtained. The distances from the tandem to the pubic symphysis and both balloons to stable bony landmarks, including the pubic symphysis, right, and left femoral heads, were measured on both paired scans, and the overnight change was calculated. Comparisons of the volumes of the anterior and posterior balloons were also made between the first and second CT simulations for the same $\mathrm{T}+\mathrm{O}$ implant to evaluate for volume loss overnight.

Sim 2 underwent retrospective dosimetric planning, and radiation to OARs (bladder and rectum) were measured in $\mathrm{D}_{2 \mathrm{cc}}, \mathrm{D}_{1 \mathrm{cc},}, \mathrm{D}_{0.1 \mathrm{cc}}$ and $\mathrm{D}_{0.03 \mathrm{cc}}$. These values were then compared to the initial values calculated from the $\operatorname{sim} 1$ treatment plan. 
Table 1. Mean volumes of anterior and posterior balloons with mean difference and percent change with standard deviation after balloons left in situ overnight

\begin{tabular}{lccccc} 
Balloon volume & Volume before $(\mathrm{cc})$ & Volume after (cc) & $\begin{array}{c}\text { Mean absolute } \\
\text { change }(\mathrm{cc})\end{array}$ & Percent change (\%) & $P$-value \\
\hline Anterior balloon & $46.84 \pm 8.61$ & $45.08 \pm 7.84$ & $-1.75 \pm 4.33$ & $-2.85 \pm 10.94$ & 0.38 \\
\hline Posterior balloon & $36.24 \pm 9.48$ & $36.75 \pm 10.01$ & $0.51 \pm 3.14$ & $1.19 \pm 9.72$ & 0.99
\end{tabular}

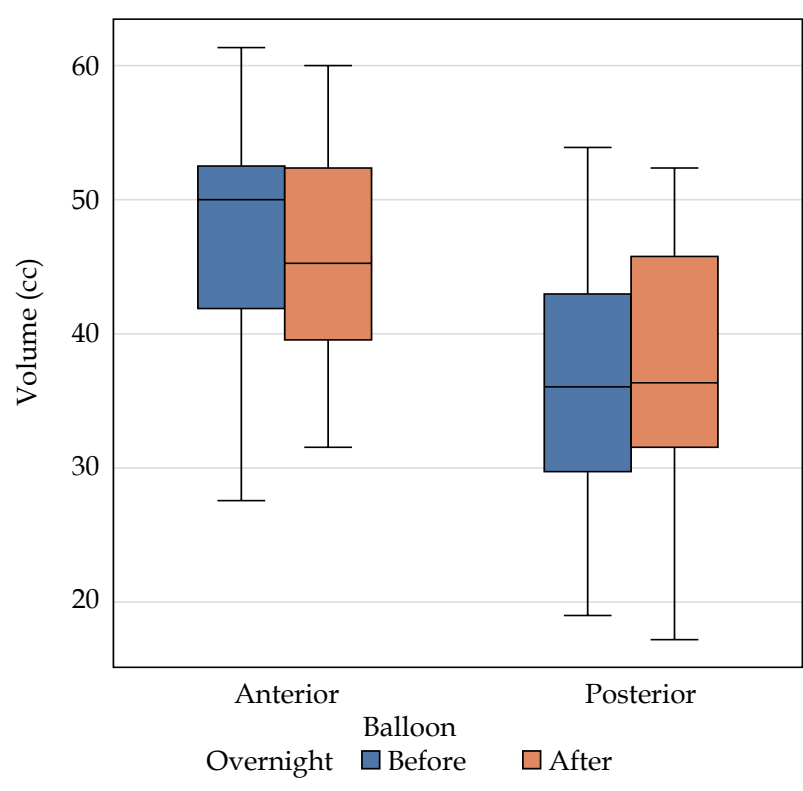

Fig. 1. Volume of balloons overnight. A box-and-whisker plot comparison of the volumes of anterior and posterior balloons before ( $\operatorname{sim} 1)$ and after (sim 2$)$ overnight stay at the hospital, with balloons and $\mathrm{T}+\mathrm{O}$ applicator remaining in situ

\section{Statistics}

The mean difference in overnight change of the balloon volumes and bony landmark measurements for the
21 treatments were reported with standard deviation, and statistical significance was calculated comparing both means using Mann-Whitney $U$ test. Statistical significance was considered $p<0.05$.

\section{Results}

\section{Balloon volumes}

The measurements of the balloon volumes were taken before the first fraction $(\operatorname{sim} 1)$ and second fraction $(\operatorname{sim} 2)$ to ascertain overnight change (Table 1, Figure 1). There was no statistically significant difference in volumes for the posterior or anterior balloon.

\section{Balloon positioning}

The change in distance from balloons and tandem to bony landmarks was calculated from the paired CT scans (Table 2, Figure 2). The anterior balloon showed no significant difference in distance from the right and left femoral heads or from the pubic symphysis. The posterior balloon had no significant difference in distance from the left femoral head, but there was a significant difference from the pubic symphysis and right femoral head. Differences from the posterior balloon to the pubic symphysis and right femoral head were $-0.29 \pm 0.46 \mathrm{~cm}(p=0.03)$ and $-0.32 \pm 0.50 \mathrm{~cm}(p=0.01)$, respectively. The tandem to the pubic symphysis also showed a significant difference, with a difference of $-0.37 \pm 0.39 \mathrm{~cm}(p=0.002)$.

Table 2. Mean distances between balloons, tandem, and bony landmarks from before (sim 1$)$ and after ( $\operatorname{sim} 2)$ overnight stay in the hospital, mean difference in distances from $\operatorname{sim} 1$ and $\operatorname{sim} 2$ overnight, and percent change with standard deviation. Bolded numbers indicate statistical significance with Mann-Whitney $U$ test

\begin{tabular}{|c|c|c|c|c|c|c|}
\hline Landmark A & Landmark B & $\begin{array}{c}\text { Distance } \\
\text { before }(\mathrm{cm})\end{array}$ & $\begin{array}{l}\text { Distance } \\
\text { after }(\mathrm{cm})\end{array}$ & $\begin{array}{l}\text { Mean difference } \\
(\mathrm{cm})\end{array}$ & $\begin{array}{c}\text { Percent change } \\
(\%)\end{array}$ & $P$-value \\
\hline \multirow[t]{3}{*}{ Anterior balloon } & Pubic symphysis & $2.12 \pm 0.38$ & $2.00 \pm 0.30$ & $-0.12 \pm 0.33$ & $-3.93 \pm 16.48$ & 0.07 \\
\hline & Right femoral head & $6.19 \pm 1.14$ & $5.87 \pm 1.24$ & $-0.33 \pm 0.76$ & $-5.06 \pm 11.22$ & 0.66 \\
\hline & Left femoral head & $6.03 \pm 1.26$ & $5.93 \pm 1.23$ & $-0.09 \pm 1.05$ & $-0.05 \pm 17.70$ & 0.66 \\
\hline \multirow[t]{3}{*}{ Posterior balloon } & Pubic symphysis & $4.90 \pm 0.57$ & $4.61 \pm 0.56$ & $-0.29 \pm 0.46$ & $-5.51 \pm 8.92$ & 0.03 \\
\hline & Right femoral head & $6.06 \pm 0.74$ & $5.74 \pm 0.64$ & $-0.32 \pm 0.50$ & $-4.95 \pm 6.94$ & 0.01 \\
\hline & Left Femoral Head & $6.32 \pm 0.59$ & $6.23 \pm 0.69$ & $-0.09 \pm 0.60$ & $-1.13 \pm 8.79$ & 0.99 \\
\hline Tandem & Pubic symphysis & $6.99 \pm 0.70$ & $6.61 \pm 0.78$ & $-0.37 \pm 0.39$ & $-5.38 \pm 5.81$ & 0.002 \\
\hline
\end{tabular}



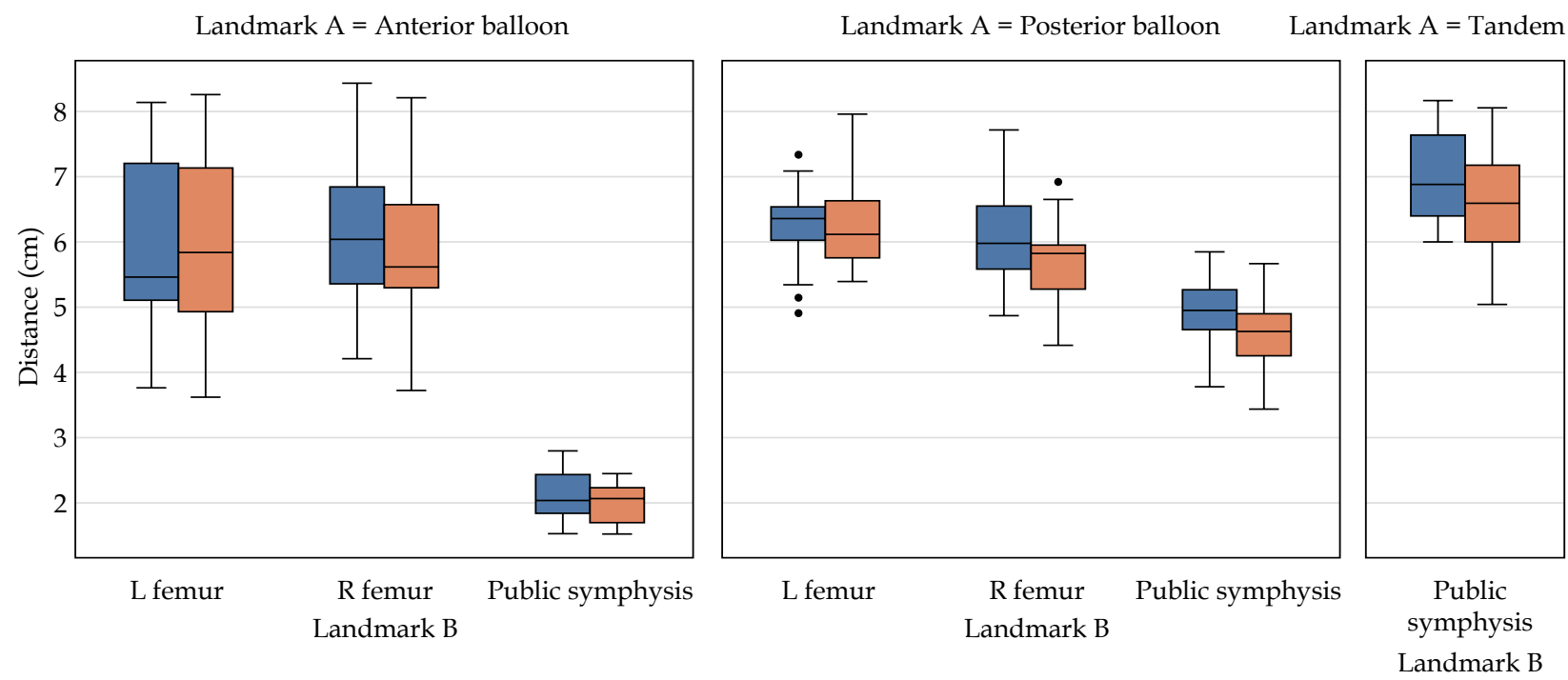

Overnight

$\square$ Before

$\square$ After

Fig. 2. Distances between bony landmarks and balloons. A box-and-whisker plot comparison of distances between bony landmarks and anterior balloon, posterior balloon, and tandem with overnight change in distances between balloons and bone (femur indicates femoral head), with balloons and $\mathrm{T}+\mathrm{O}$ applicator in situ

\section{Dosimetric changes}

After analysis of the data of the 21 paired scans with balloons remaining inflated in situ, there was no significant differences in the bladder and rectum radiation doses (Table 3, Figure 3).

\section{Discussion}

Brachytherapy is an important tool in the definitive treatment of cervical cancer $[5,6]$. Patients in rural areas who live a significant distance away from a brachytherapy center, face inconvenience with multiple procedures for $\mathrm{T}+\mathrm{O}$ HDR brachytherapy delivery. Brachytherapy is a highly specialized treatment that can be difficult to access in rural parts of high-income countries, particularly for older women [17], and also in low- and middle-income countries, where up to $90 \%$ of cervical cancer patients may not have access to brachytherapy [18].

For many patients though, there are numerous access barriers to treatment, such as lack of transportation and support $[19,20]$. Treatment over sequential days after completion of chemoradiation to the pelvis, allows patients to expedite their overall brachytherapy treatment during an overnight stay at the hospital, thus easing a considerable travel burden for rural patients coming to a brachytherapy center.

There are numerous ways to displace OARs during brachytherapy procedures, including traditional gauze packing, balloons, and retractor blades [12]. Evaluating the overnight variability of these devices is important for do-

Table 3. Mean radiation dose to organs at risk at first dose (sim 1) and second dose (sim 2), mean difference in radiation dose between $\operatorname{sim} 1$ and $\operatorname{sim} 2$, and percent change with standard deviation

\begin{tabular}{|c|c|c|c|c|c|}
\hline Organ at risk & $\begin{array}{c}\text { Mean first dose } \\
\text { (cGy) }\end{array}$ & $\begin{array}{l}\text { Mean second dose } \\
\text { (cGy) }\end{array}$ & $\begin{array}{c}\text { Mean difference } \\
\text { (cGy) }\end{array}$ & $\begin{array}{l}\text { Percent change } \\
\text { (\%) }\end{array}$ & $P$-value \\
\hline \multicolumn{6}{|l|}{ Bladder } \\
\hline $\mathrm{D}_{0.03 c c}$ & $1427.67 \pm 790.61$ & $2027.88 \pm 1881.82$ & $600.21 \pm 1917.84$ & $56.62 \pm 148.92$ & 0.66 \\
\hline $\mathrm{D}_{0.1 c c}$ & $1279.66 \pm 639.99$ & $1735.01 \pm 1519.08$ & $455.34 \pm 1503.40$ & $44.14 \pm 124.76$ & 0.99 \\
\hline $\mathrm{D}_{1 \mathrm{cc}}$ & $952.81 \pm 380.54$ & $1139.89 \pm 837.49$ & $187.08 \pm 716.92$ & $18.24 \pm 69.57$ & 0.99 \\
\hline $\mathrm{D}_{2 \mathrm{cc}}$ & $850.83 \pm 328.60$ & $969.22 \pm 661.22$ & $118.39 \pm 523.75$ & $11.33 \pm 55.22$ & 0.99 \\
\hline \multicolumn{6}{|l|}{ Rectum } \\
\hline$\overline{D_{0.03 c c}}$ & $593.75 \pm 341.84$ & $596.35 \pm 308.50$ & $2.59 \pm 196.82$ & $16.91 \pm 92.166$ & 0.66 \\
\hline $\mathrm{D}_{0.1 c c}$ & $558.86 \pm 303.79$ & $565.06 \pm 288.11$ & $6.20 \pm 168.39$ & $15.87 \pm 87.57$ & 0.65 \\
\hline $\mathrm{D}_{1 \mathrm{cc}}$ & $468.77 \pm 230.02$ & $467.07 \pm 221.21$ & $-1.70 \pm 111.46$ & $11.20 \pm 72.49$ & 0.38 \\
\hline$D_{2 c c}$ & $432.90 \pm 206.27$ & $426.12 \pm 196.67$ & $-6.79 \pm 94.74$ & $8.97 \pm 66.63$ & 0.38 \\
\hline
\end{tabular}




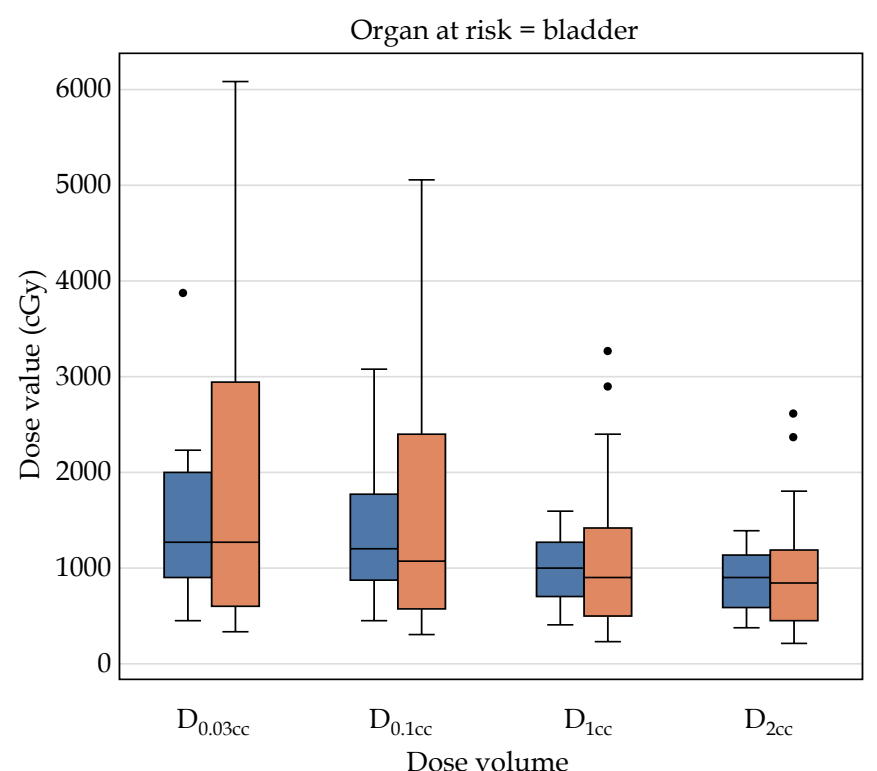

Overnight $\square$ Before

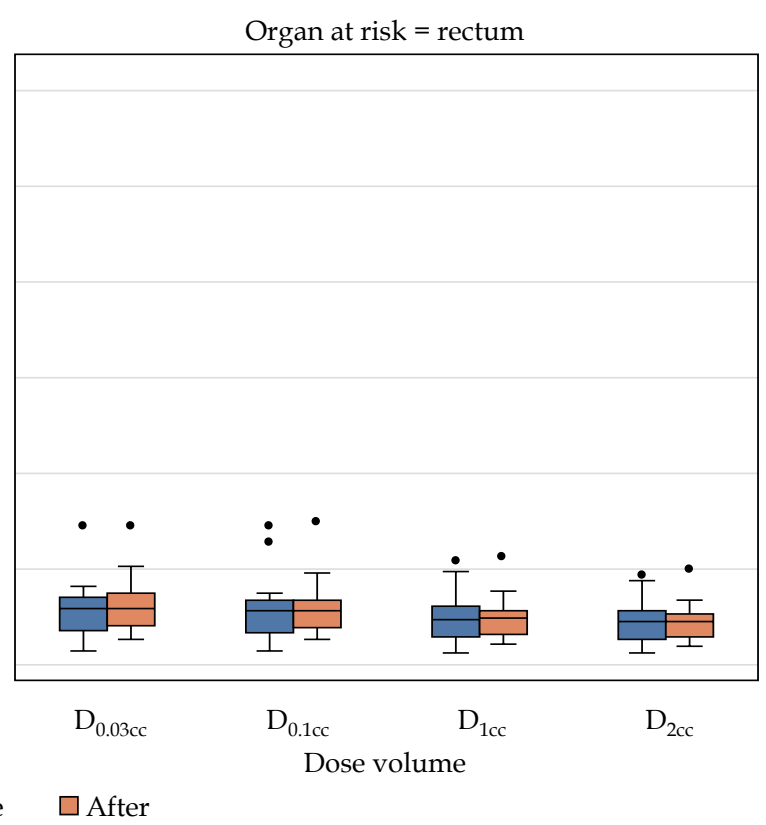

$\square$ After

Fig. 3. Organs at risk and dose. A box-and-whisker plot comparison of radiation dose delivered to bladder and rectum before $(\operatorname{sim} 1)$ and after $(\operatorname{sim} 2)$ overnight stay at the hospital, with balloons and $\mathrm{T}+\mathrm{O}$ applicator remaining in situ

simetric reasons and disease control, as Viswanathan et al. [21] showing that variability in brachytherapy placement may decrease disease control. As air-filled balloons are an increasingly common packing method [22], it is important to understand the implications of overnight movements and intracavitary pressures on the balloons and $\mathrm{T}+\mathrm{O}$ applicator. Moreover, it is crucial to ensure that any resultant movements do not compromise the dose and cause potential complications to the bladder and rectum.

The purpose of this study was to assess whether overnight shifting and external pressure (forces external to the balloon, such as applied by body tissues, including the musculature of the vagina that could change the shape, position, or cause deflation of the balloon) while the patient had the $\mathrm{T}+\mathrm{O}$ and balloons in situ for over 24 hours, resulted in significant changes in balloon dimensions, balloon positioning, and $\mathrm{T}+\mathrm{O}$ positioning. There were no statistically significant changes in the volume of either the anterior or posterior balloons overnight. Positioning was measured in relation to unchanging bony landmarks. There were no significant changes found in the positioning of the anterior balloon. The mean changes in position of the posterior balloon and tandem were below $0.5 \mathrm{~cm}$, and our data did not show any resultant increase in the bladder or rectum dose between CT sim 1 and CT sim 2. Although the distance between the posterior balloon and pubic symphysis did change significantly overnight in relation to certain bony landmarks, this did not appear to result in a dosimetric effect. Presumably, the target and OARs moved with the applicator/balloons, and so did not alter the radiation dose to those structures.

Despite potential human error with contouring of balloons and measurements, this data supports the use of vaginal immobilization balloons as a potential alternative to gauze packing for selected patients' populations, showing minimal or no adverse positioning or radiation effects from keeping the $\mathrm{T}+\mathrm{O}$ applicator in situ overnight.

\section{Conclusions}

While this study is limited in its capacity as a retrospective analysis of patients, in whom this treatment was performed, given the therapy's potential logistical benefit, this data merits further prospective study of the sequential day treatment regimen using vaginal immobilization balloons. These data can add to our understanding of alternative brachytherapy techniques to better serve our patients.

\section{Acknowledgments}

We would like to acknowledge UACC Better Than Ever program funding.

\section{Disclosure}

The authors report no conflict of interest.

\section{References}

1. Arbyn M, Weiderpass E, Bruni L et al. Estimates of incidence and mortality of cervical cancer in 2018: a worldwide analysis. Lancet Glob Health 2020; 8: e191-203.

2. Guo F, Cofie LE, Berenson AB. Cervical cancer Incidence in young U.S. females after human papillomavirus vaccine introduction. Am J Prev Med 2018; 55: 197-204.

3. Practice Bulletin No. 168: Cervical Cancer Screening and Prevention. Obstet Gynecol 2016; 128: e111-130.

4. Cho O, Chun M. Management for locally advanced cervical cancer: new trends and controversial issues. Radiat Oncol J 2018; 36: 254-264.

5. Gursel SB, Serarsian A, Meydan AD et al. A comparison of tandem ring and tandem ovoid treatment as a curative brachytherapy component for cervical cancer. J Contemp 
Brachytherapy 2020; 12: 111-117.

6. Viswanathan AN, Beriwal S, De Los Santos JF et al. American Brachytherapy Society consensus guidelines for locally advanced carcinoma of the cervix. Part II: high-dose-rate brachytherapy. Brachytherapy 2012; 11: 47-52.

7. Gill BS, Lin JF, Krivak TC et al. National cancer data base analysis of radiation therapy consolidation modality for cervical cancer: the impact of new technological advancements. Int J Radiat Oncol Biol Phys 2014; 90: P1083-1090.

8. Tanderup C, Eifel PJ, Yashar CM et al. Curative radiation therapy for locally advanced cervical cancer: brachytherapy is NOT optional. Int J Radiat Oncol Biol Phys 2013; 88: P537-539.

9. Viswanathan AN, Thomadsen B, American Brachytherapy Society Cervical Cancer Recommendations. American brachytherapy society consensus guidelines for locally advanced carcinoma of cervix. Part I: general principles. Brachytherapy 2012; 11: 33-46.

10. Banerjee R, Kamrava M. Brachytherapy in the treatment of cervical cancer: a review. Int J Womens Health 2014; 6: 555-564.

11. Montana GS, Fowler WC. Carcinoma of the cervix: analysis of bladder and rectal radiation dose and complications. Int J Radiat Oncol Biol Phys 1989; 16: P95-100.

12. Sud S, Roth T, Jones E. Clinical analysis of speculum-based vaginal packing for high-dose-rate intracavitary tandem and ovoid brachytherapy in cervical cancer. J Contemp Brachytherapy 2018; 10: 32-39.

13. Malaker $\mathrm{K}$, Shukla V, D'Souza $\mathrm{H}$ et al. Minimizing urinary bladder radiation dose during brachytherapy for carcinoma of the cervix using balloon inflation technique. Int J Radiat Oncol Biol Phys 2005; 61: 257-266.

14. Eng TY, Patel AJ, Ha CS. Rectal and bladder dose reduction with the addition of intravaginal balloons to vaginal packing in intracavitary brachytherapy for cervical cancer. Brachytherapy 2016; 15: 312-318.

15. Rai B, Patel FD, Chakraborty S et al. Bladder-rectum spacer balloon in high-dose-rate brachytherapy in cervix carcinoma. Int J Radiat Oncol Biol Phys 2013; 85: e217-222.

16. Goyal U, Pan J, Dougherty S. Immobilization balloons used for specialized brachytherapy treatments for cervical cancer patients. Brachytherapy 2016; 15: S110-S111.

17. Spees LP, Wheeler SB, Varia M et al. Evaluating the urban-rural paradox: the complicated relationship between distance and the receipt of guideline-concordant care among cervical cancer patients. Gynecol Oncol 2019; 152: P112-118.

18. Grover S, Longo J, Einck J et al. The unique issues with brachytherapy in low- and middle-income countries. Semin Radiat Oncol 2017; 27: 136-142.

19. Ramondetta LM, Meyer LA, Schmeler KM et al. Avoidable tragedies: disparities in healthcare access among medically underserved women diagnosed with cervical cancer. Gynecol Oncol 2015; 139: 500-555.

20. Ramondetta LM, Sun C, Hollier L et al. Advanced cervical cancer treatment in Harris county: pilot evaluation of factors that prevent optimal therapy. Gynecol Oncol 2006; 103: 547-553.

21. Viswanathan AN, Moughan J, Small W et al. The quality of cervical cancer brachytherapy implantation and the impact on local recurrence and disease-free survival in radiation therapy oncology group prospective trials 0116 and 0128 . Int J Gynecol Cancer 2012; 22: 123-131.

22. Xu-Welliver M, Lin LL. Evaluation of a balloon-based vaginal packing system and patient-controlled analgesia for patients with cervical cancer undergoing high-dose-rate intracavitary brachytherapy. Pract Radiat Oncol 2012; 3: P263-268. 